\title{
Efficacy of acoustic radiation force impulse elastography in differentiation of benign and malignant lymph nodes
}

\begin{abstract}
Aims and Objectives: Aim of this study was to assess the clinical usefulness of Acoustic Radiation Force Impulse (ARFI) elastography imaging in differentiating benign and malignant/metastatic superficial nodes.

Methods and Materials: ARFI elastography (Siemens Medical Solutions, Mountain View, CA) using Virtual touch Tissue Imaging (VTI) technique was performed and Shear wave velocity $(\mathrm{m} / \mathrm{sec})$ was measured in 85 patients (mean age, 49 years; range, 16-72 years) with 126 lymph nodes (96 axillary, 21 cervical and 9 inguinal nodes) which were biopsied and histo-pathologically correlated.

Results: Of 126 nodes, 71 were benign (64 reactive and 7 koch's) and 55 were malignant (40 metastases and 15 lymphoma). The mean ARFI value of malignant nodes $\pm S D(4.42 \pm 2.60)$ were significantly higher than the benign nodes $(1.76 \pm 0.44)$, with $P<0.001$. With the use of Receiver Operating Characteristic (ROC) curve, the cut off SWV was estimated to be $2.45 \mathrm{~m} / \mathrm{s}$ with sensitivity of $83.6 \%$, specificity of $91 \%$, and accuracy of $93.7 \%$ while the conventional US based short axis diameter (SAD) measurement with cut off value of $10 \mathrm{~mm}$, showed the sensitivity of $62 \%$, specificity of $87 \%$ and accuracy of $87 \%$.

Conclusion: Acoustic radiation force impulse elastography is feasible for superficial lymph nodes and provides quantitative elasticity measurements, which may complement B-mode ultrasound and potentially improve the differentiation of benign and malignant/metastatic lymph nodes.
\end{abstract}

Keywords: Acoustic radiation force impulse elastography, benign, lymph nodes, malignant, shear wave, ultrasound

\section{INTRODUCTION}

An enlarged lymph node in the body can be due to reactive hyperplasia, granulomatous or nongranulomatous lymphadenitis, lymphoma or metastasis. Identification of malignant or metastatic lymph node is extremely important because it helps in the diagnosis, nodal staging of a known malignant disease, prognosis, and determination of optimal therapeutic options. Conventional ultrasonography (US) is an effective method for identifying abnormal lymph nodes based on gray-scale and color Doppler imaging. However, the US criteria for detecting malignant or metastatic nodes are still controversial. ${ }^{[1-3]}$

Sonoelastography is a promising US-based imaging modality, which is being used to assess the stiffness of the tissue examined. Strain elastography has been used to assess the elasticity of various organs including lymph nodes, but it is a qualitative or semiquantitative way of assessing elasticity with freehand compression technology. Acoustic radiation

\begin{tabular}{|l|c|}
\hline \multicolumn{2}{|c|}{ Access this article online } \\
Website: & Quick Response Code \\
www.asjo.in & \\
\hline &
\end{tabular}

force impulse (ARFI) is a recent US-based technology, which noninvasively provides numeric value of tissue stiffness without applying external compression. Hence, it is a less operator-dependent method of assessing stiffness of the tissue. In this imaging method, a specially designed transducer is used, which produces focused acoustic impulses of high intensity, called push pulses, which are transmitted to the tissue and causing shear wave formation within the region of interest. The velocities of the induced shear

\section{Rashmi Sudhir, M. V. T. KRIShna MOHan ${ }^{1}$, Bogala Shilpa, Veeraiah K. Chaudhary}

Department of Radiodiagnosis and ${ }^{1}$ Department of Medical Oncology, Basavatarakam Indo American Cancer Hospital and Research Institute, Hyderabad, Telangana, India

Address for correspondence: Dr. Rashmi Sudhir, Flat No 205, Yashoda Brindavan, HUDA Enclave, Jubilee Hills, Hyderabad - 500 096, Telangana, India.

E-mail: rashmi4210@gmail.com

This is an open access article distributed under the terms of the Creative Commons Attribution-NonCommercial-ShareAlike 3.0 License, which allows others to remix, tweak, and build upon the work non-commercially, as long as the author is credited and the new creations are licensed under the identical terms.

For reprints contact: reprints@medknow.com

How to cite this article: Sudhir R, Krishna Mohan MV, Shilpa B, Chaudhary VK. Efficacy of acoustic radiation force impulse elastography in differentiation of benign and malignant lymph nodes. Asian $\mathrm{J}$ Oncol 2017;3:106-10. 
waves are tracked by the ARFI imaging using ultrasound correlation methods. The velocity of shear wave propagation is proportional to the square root of the tissue elasticity, based on Young's modulus formula: $\mathrm{E}=3 \rho \mathrm{c} 2$ where $\mathrm{E}$ is stiffness $(\mathrm{kPa}), \mathrm{c}$ is the shear wave velocity $(\mathrm{SWV})(\mathrm{m} / \mathrm{s})$, and $\rho$ is the density $\left(\mathrm{kg} / \mathrm{m}^{3}\right)$ of the tissue. The density of the soft tissue is almost same as the density of water $\left(1000 \mathrm{~kg} / \mathrm{m}^{3}\right)$. Thus, by measuring SWV, tissue stiffness is measured quantitatively. ${ }^{[4]}$ ARFI elastography based on SWV measurement has been used earlier for many other organs, for example, breast, thyroid, liver, prostate, kidney, spleen, and pancreas. ${ }^{\mid 5-12]}$

To the best of our knowledge, very few studies have been done to evaluate the diagnostic performance of ARFI elastography for cervical nodes, ${ }^{[12]}$ and yet, there is no study done including all superficial nodes (cervical, axillary and inguinal nodes) of different histopathologies. Hence, this study was done to assess the clinical usefulness of ARFI imaging in differentiation of benign and malignant/metastatic superficial lymph nodes of various histopathologies.

\section{MATERIALS AND METHODS}

This is a prospective study which was carried out to assess the clinical usefulness of ARFI elastography imaging in differentiating benign and malignant/metastatic lymph nodes. One hundred consecutive patients who underwent conventional US and ARFI elastography evaluation followed by pathological diagnosis of cervical, axillary, or inguinal nodes at our center between January 2015 and September 2015 were enrolled in this study. Lymph nodes with at least one abnormal sonographic feature, which were sent for fine needle aspiration or core biopsy or underwent excision biopsy, were included in this study. Ten patients who previously underwent chemotherapy were excluded, and 5 patients were excluded due to lack of histopathological correlation. So, finally, a total of 85 patients with 126 nodes were included in this study.

Image acquisition and measurement of shear wave velocity Both conventional US and ARFI elastography using virtual touch tissue quantification (VTQ) technique were performed using Siemens ACUSON S2000 US system using linear array, 9 L4 transducer with bandwidth of 4-9 MHz. On conventional US, size (short axis diameter [SAD]), short- and long-axis diameter ratio, presence or loss of fatty hilum, presence of calcification of necrosis or calcification, and cortical thickness of each node were recorded. For ARFI imaging, the transducer was applied with the minimal pressure just enough to make a complete contact with the node without any breath hold. On B-mode US, the area within the node to be evaluated for its elastic properties was defined by a fixed box measuring $10 \mathrm{~mm} \times 6 \mathrm{~mm}$ [Figure 1].

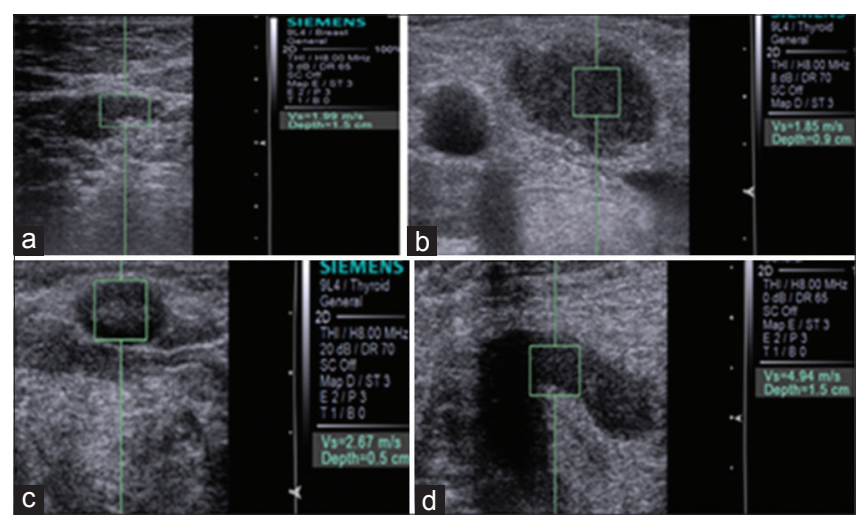

Figure 1: (a) Case of carcinoma breast with ipsilateral axillary node, $20 \mathrm{~mm} \times 10 \mathrm{~mm}$ with thickened cortex, acoustic radiation force impulse revealed shear wave velocity of $1.99 \mathrm{~m} / \mathrm{s}$ and biopsy suggested reactive hyperplasia. (b) Cervical node, $20 \mathrm{~mm} \times 13 \mathrm{~mm}$ with loss of fatty hilum, acoustic radiation force impulse showed shear wave velocity of $1.85 \mathrm{~m} / \mathrm{s}$ and biopsy suggested tuberculous lymphadenitis. (c) Submental node, $11 \mathrm{~mm} \times 5 \mathrm{~mm}$ with preserved fatty hilum, acoustic radiation force impulse image showed shear wave velocity of $2.67 \mathrm{~m} / \mathrm{s}$. and biopsy suggested Hodgkin's lymphoma. (d) Case of carcinoma breast with axillary node, $26 \mathrm{~mm} \times 18 \mathrm{~mm}$ with thickened cortex, acoustic radiation force impulse showed shear wave velocity of $4.94 \mathrm{~m} / \mathrm{s}$ and biopsy revealed metastases from ductal carcinoma breast

Three measurements were obtained from each node, and the mean with standard deviation was calculated. All US examinations were performed by two experienced radiologists with more than 7 years of experience in sonomammography and adequate training in performing elastography.

\section{Pathological diagnoses}

All lymph nodes included in this study were pathologically examined and correlated with either fine needle aspiration cytology or histopathology of core biopsy or excision biopsy sample. All pathological examinations were done by experienced pathologists.

\section{Statistical analysis}

The mean SWV of malignant and benign lymph nodes were compared with unpaired Student's $t$-test. The receiving operating characteristic (ROC) curve analysis was done to measure and compare the efficacy of SWV and SAD in differentiating malignant from benign node. The best cutoff was obtained by Youden index (sensitivity + specificity-1) from ROC curve analysis. The sensitivity and specificity values with $95 \%$ confidence interval were estimated. $P<0.05$ was taken as statistical significant. All data were analyzed using SPSS software 20 version for Windows.

\section{RESULTS}

There were 126 nodes from 85 patients with mean age 49 years (age range 18-72 years) included in this study, of which 71 were benign (64 reactive hyperplasia and 7 tuberculous) and 
55 were malignant (40 metastases and 15 lymphoma) [Table 1]. ARFI images with SWV of various histopathologies are shown in Figure 1a-d.

On ARFI imaging, the SWV of malignant nodes ranged from 1.3 to $9.1 \mathrm{~m} / \mathrm{s}$ (mean $4.42 \pm 2.60 \mathrm{~m} / \mathrm{s}$ ) was significantly higher than the benign nodes with SWV ranging from 0.8 to $3 \mathrm{~m} / \mathrm{s}$ (mean $1.76 \pm 0.44 \mathrm{~m} / \mathrm{s}$ ), with $P<0.001$ [Figure 2].

With the ROC curve analysis, the best cutoff value of the malignant nodes from benign nodes was estimated to be $2.45 \mathrm{~m} / \mathrm{s}$ with sensitivity of $83.6 \%$, specificity of $91 \%$, and area under the curve (AUC) equal to 0.937, whereas with the conventional US-based SAD measurement, with cutoff value of $10 \mathrm{~mm}$, the sensitivity was $62 \%$, specificity $87 \%$, and AUC 0.870 [Figures 3 and 4].

There was no significant difference in the SWV of reactive and granulomatous nodes which were between $0.8-2.6 \mathrm{~m} / \mathrm{s}$ (mean $1.72 \pm 0.4 \mathrm{~m} / \mathrm{s}$ ) and $1.5-3 \mathrm{~m} / \mathrm{s}$ (mean $2.12 \pm 0.59 \mathrm{~m} / \mathrm{s}$ ), respectively. The different histopathological features of malignant nodes also did not show significant difference which ranged between $1.3-9.1 \mathrm{~m} / \mathrm{s}(4.1 \pm 2.45 \mathrm{~m} / \mathrm{s})$ for lymphoma and $1.5-9.1 \mathrm{~m} / \mathrm{s}(4.54 \pm 2.67 \mathrm{~m} / \mathrm{s})$ for metastatic nodes [Figure 5].

\section{DISCUSSION}

In last few years, conventional elastography (strain or real-time elastography) has shown promising results for measuring the stiffness of the tissue inside a region of interest. However, the measurement methods of conventional elastography were semiquantitative in which the stiffness was given in the form of scores and patterns after applying appropriate amount of mechanical pressure on the tissue of interest through transducer which is highly operator

Table 1: Pathological diagnoses of lymph nodes $(n=126)$

\begin{tabular}{|c|l|}
\hline Diagnosis & N \\
\hline Benign nodes & $\mathbf{7 1}$ \\
\hline Reactive hyperplasia & 64 \\
\hline Tuberculosis & 07 \\
\hline Malignant nodes & $\mathbf{5 5}$ \\
\hline Metastases & 40 \\
\hline Lymphoma & 15 \\
\hline
\end{tabular}

dependent and did not show consistent results in various studies. ${ }^{[13,14]}$

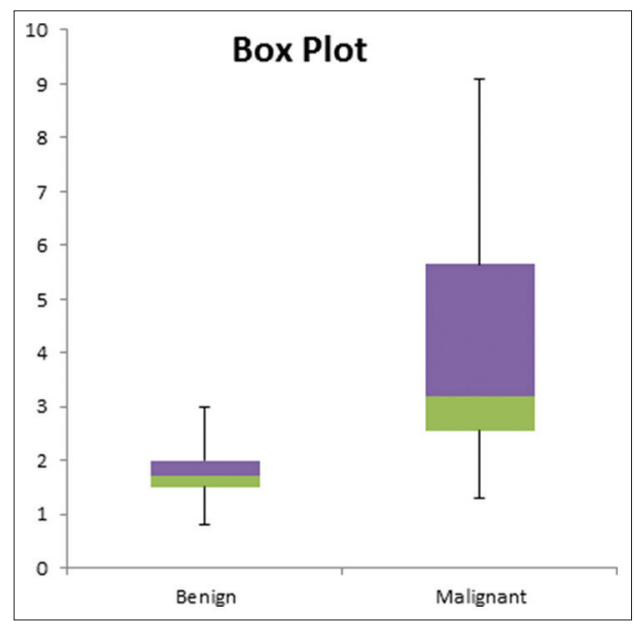

Figure 2: Mean shear wave velocities of benign and malignant nodes

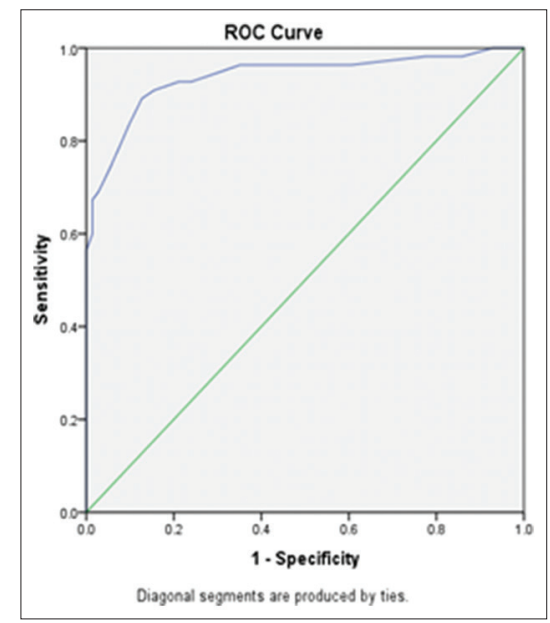

Figure 3: Receiving operating characteristic curve to estimate the cutoff value of acoustic radiation force impulse to detect malignant nodes

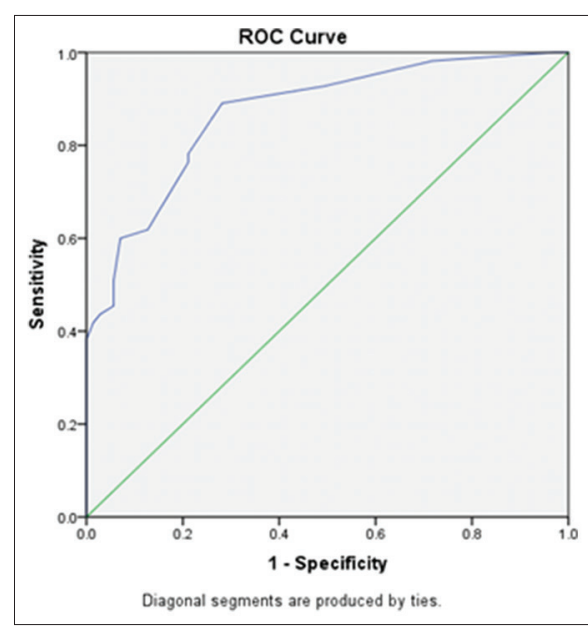

Figure 4: Receiving operating characteristic curve for conventional ultrasonography based on short axis diameter of lymph node 


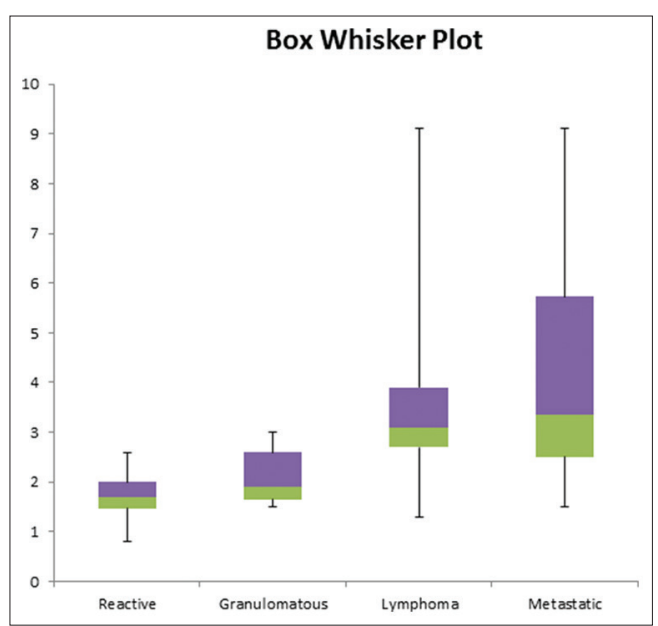

Figure 5: Shear wave velocities of different histopathological lymph nodes

ARFI is a relatively new imaging method which uses the VTQ technique to measure the velocity of horizontal shear wave induced in the region of interest after sending longitudinal acoustic push pulse from the transducer. The faster the shear wave propagates, the harder is the tissue. VTQ technique does not rely on the operator's skills to apply appropriate amount of mechanical pressure to the target tissue. It provides more accurate elasticity of the nodes as it does not get affected by the surrounding hard or soft areas in the vicinity allowing better assessment of the mechanical properties of the tissue than the conventional elastography where entire tissue in the region of interest moves simultaneously under the external stress applied through the transducer.

In general, malignant lymph nodes are stiffer than the benign nodes due to the biological characteristic of tumor infiltration. ${ }^{[13-18]}$ In a recent meta-analysis done by Zhang et al., discriminated 1084 benign and malignant lymph nodes from 9 studies based on ARFI cutoff values with pooled sensitivity, specificity, and AUC were $0.87,0.88$, and 0.93 , respectively. In this meta-analysis, majority of the studies were included from China and one each from Japan, South Korea, and USA. ${ }^{[19]}$ However, there are no studies done yet from India. In our study, ARFI imaging method revealed significantly higher mean SWV of malignant nodes (lymphoma and metastases) as compared to benign nodes [Figure 3]. With the cutoff SWV value of $2.45 \mathrm{~m} / \mathrm{s}$, the sensitivity and specificity were $83.6 \%$ and $91 \%$, respectively, with area under the ROC curve was 0.937 . Our study also revealed that there was a significant difference between the cutoff SWV value of tuberculous nodes (mean SWV $2.13 \mathrm{~m} / \mathrm{s}$ ) and lymphomatous nodes (mean SWV $4.10 \mathrm{~m} / \mathrm{s}$ ) though studies done by Lenghel et al., 2012 revealed that lymphomatous nodes are softer nodes and Teng et al., 2012 showed that tuberculous lymph nodes may show stiffer areas or heterogeneous stiffness due to fibrosis, calcification, or necrosis. Similar to studies done by
Bhatia et al., 2012 and Choi et al., 2015, we also found that tuberculous nodes were stiffer than the reactive nodes (mean SWV $1.72 \mathrm{~m} / \mathrm{s}$ ), and metastatic nodes (mean SWV $4.54 \mathrm{~m} / \mathrm{s}$ ) were the hardest nodes in comparison to the reactive, tuberculous, and lymphomatous nodes. In this study, all lymph nodes with SWV of $<1.3 \mathrm{~m} / \mathrm{s}$ were found to be nonmalignant suggesting that ARFI imaging is very useful in excluding malignant or metastatic nodes. Fujiwara et al., 2013 found negative likelihood ratio of 0.06 , and all lymph nodes with $\mathrm{SWV}<1.4 \mathrm{~m} / \mathrm{s}$ were found to be reactive; thus, it may be very useful in reducing the number of unnecessary nodal biopsies or dissection.

\section{Limitations}

There are few limitations with this study. First, there are still no clear-cut guidelines regarding placement of ROI within the node and number of values to be taken for the calculation of mean SWV. Second, nodes with calcification or necrosis may give false values. Third, distance of the node from the transducer and intervening different tissues may produce variable results. Fourth, there is no clear cutoff SWV values for different manufacturing US machines, even different machines used of same manufacturing company give slightly different cutoff values for the same organ. Fifth, metastatic nodes are not specified in this study to compare the SUV values of different histopathological primaries.

\section{CONCLUSION}

ARFI elastography is a promising noninvasive technique which further increases the specificity of US in differentiation of benign and malignant/metastatic lymph nodes and thus helps in selecting the most appropriate lymph node to biopsy in a given clinical situation and also in reducing the number of unnecessary nodal biopsies and excisions.

Financial support and sponsorship

Nil.

\section{Conflicts of interest}

There are no conflicts of interest.

\section{REFERENCES}

1. Giacomini CP, Jeffrey RB, Shin LK. Ultrasonographic evaluation of malignant and normal cervical lymph nodes. Semin Ultrasound CT MR 2013;34:236-47.

2. Lyshchik A, Higashi T, Asato R, Tanaka S, Ito J, Hiraoka M, et al. Cervical lymph node metastases: Diagnosis at sonoelastography - Initial experience. Radiology 2007;243:258-67.

3. Meng W, Zhang G, Wu C, Wu G, Song Y, Lu Z. Preliminary results of acoustic radiation force impulse (ARFI) ultrasound imaging of breast lesions. Ultrasound Med Biol 2011;37:1436-1443.

4. Palmeri ML, Nightingale KR. Acoustic radiation force-based elasticity 
imaging methods. Interface Focus 2011;1:553-64.

5. Bojunga J, Dauth N, Berner C, Meyer G, Holzer K, Voelkl L, et al. Acoustic radiation force impulse imaging for differentiation of thyroid nodules. PLoS One 2012;7:e42735.

6. Zhang FJ, Han RL. The value of acoustic radiation force impulse (ARFI) in the differential diagnosis of thyroid nodules. Eur J Radiol 2013;82:e686-90.

7. Tozaki M, Isobe S, Fukuma E. Preliminary study of ultrasonographic tissue quantification of the breast using the acoustic radiation force impulse (ARFI) technology. Eur J Radiol 2011;80:e182-7.

8. Zhai L, Polascik TJ, Foo WC, Rosenzweig S, Palmeri ML, Madden J, et al. Acoustic radiation force impulse imaging of human prostates: Initial in vivo demonstration. Ultrasound Med Biol 2012;38:50-61.

9. Friedrich-Rust M, Wunder K, Kriener S, Sotoudeh F, Richter S, Bojunga J, et al. Liver fibrosis in viral hepatitis: Noninvasive assessment with acoustic radiation force impulse imaging versus transient elastography. Radiology 2009;252:595-604.

10. Yoneda M, Suzuki K, Kato S, Fujita K, Nozaki Y, Hosono K, et al. Nonalcoholic fatty liver disease: US-based acoustic radiation force impulse elastography. Radiology 2010;256:640-7.

11. Lee MJ, Kim MJ, Han KH, Yoon CS. Age-related changes in liver, kidney, and spleen stiffness in healthy children measured with acoustic radiation force impulse imaging. Eur J Radiol 2013;82:e290-4.

12. Yashima Y, Sasahira N, Isayama H, Kogure H, Ikeda H, Hirano K, et al. Acoustic radiation force impulse elastography for noninvasive assessment of chronic pancreatitis. J Gastroenterol 2012;47:427-32.

13. Fujiwara T, Tomokuni J, Iwanaga K, Ooba S, Haji T. Acoustic radiation force impulse imaging for reactive and malignant/metastatic cervical lymph nodes. Ultrasound Med Biol 2013;39:1178-83.

14. Teng DK, Wang H, Lin YQ, Sui GQ, Guo F, Sun LN, et al. Value of ultrasound elastography in assessment of enlarged cervical lymph nodes. Asian Pac J Cancer Prev 2012;13:2081-5.

15. Lo WC, Liao LJ. Comparison of two elasticity scoring systems in the assessment of the cervical lymph nodes. J Med Ultrasound 2014;22:140-4.

16. Lenghel LM, Bolboaca SD, Botar-Jid C, Baciut G, Dudea SM. The value of a new score for sonoelastographic differentiation between benign and malignant cervical lymph nodes. Med Ultrason 2012;14:271-7.

17. Bhatia KS, Cho CC, Tong CS, Yuen EH, Ahuja AT. Shear wave elasticity imaging of cervical lymph nodes. Ultrasound Med Biol 2012;38:195-201.

18. Choi YJ, Lee JH, Baek JH. Ultrasound elastography for evaluation of cervical lymph nodes. Ultrasonography 2015;34:157-64.

19. Zhang P, Zhang L, Zheng S, Yu C, Xie M, Lv Q, et al. Acoustic radiation force impulse imaging for the differentiation of benign and malignant lymph nodes: A Systematic review and meta-analysis. PLoS One 2016;11:e0166716. 